\title{
Predictors of Need for Noninvasive Ventilation During Respiratory Tract Infections in Medically Stable, Non-Ventilated Subjects With Amyotrophic Lateral Sclerosis
}

\author{
Jesus Sancho MD, Emilio Servera MD, Pilar Bañuls MD, and Julio Marin MD
}

\begin{abstract}
BACKGROUND: Acute lower respiratory infections can impair muscle strength in patients with amyotrophic lateral sclerosis (ALS). When associated with an increase in load on the respiratory system, this situation may precipitate hypercapnic respiratory failure in non-ventilated patients with ALS. The aim of this study was to determine whether a clinical or functional parameter can predict the need for noninvasive ventilation (NIV) during an acute respiratory infection for medically stable, non-ventilated patients with ALS. METHODS: This was a prospective study involving all non-ventilated subjects with ALS admitted due to an acute respiratory infection to a respiratory care unit from a tertiary hospital. RESULTS: Thirty-two non-ventilated subjects with ALS were admitted to our respiratory care unit due to an acute respiratory infection: $60.72 \pm 10.54 \mathrm{y}, 13$ males, 23 with spinal onset, FVC of $1.58 \pm 0.83 \mathrm{~L}$, FVC of $56.21 \pm 23.15 \%$ of predicted, peak cough flow of $3.41 \pm 1.77 \mathrm{~L} / \mathrm{s}$, maximum insufflation capacity of $1.87 \pm 0.94 \mathrm{~L}$, revised Amyotrophic Lateral Sclerosis Functional Rating Scale score of $22.80 \pm 8.83$, and Norris bulbar score of $23.48 \pm 12.14$. Fifteen subjects required NIV during the episode. Logistic regression analysis showed that the only predictors of need for NIV were percent-of-predicted FVC (odds ratio of 1.06, 95\% CI 1.01-1.11, $P=.02$ ) and peak cough flow (odds ratio of $2.57,95 \%$ CI $1.18-5.59, P=.02$ ). CONCLUSIONS: In medically stable, non-ventilated patients with ALS, measurement of percentof-predicted FVC and peak cough flow can predict the need for NIV during an acute lower respiratory tract infection. Key words: noninvasive ventilation; respiratory failure; amyotrophic lateral sclerosis; neuromuscular disease. [Respir Care 2015;60(4):492-497. (C) 2015 Daedalus Enterprises]
\end{abstract}

\section{Introduction}

As a consequence of the natural course of amyotrophic lateral sclerosis (ALS), patients suffer progressive respiratory muscle weakness, leading to ventilatory failure and ineffective coughing, ${ }^{1}$ which are the principal causes of morbidity and mortality in these patients. ${ }^{2}$ However, during acute respiratory tract infections, an unexpected reduction in baseline respiratory muscle strength can occur, triggering acute respiratory failure. ${ }^{3,4}$

Drs Sancho, Servera, and Bañuls are affiliated with the Respiratory Care Unit, Respiratory Medicine Department, Hospital Clinico Universitario, and the Research Group for Respiratory Problems in Neuromuscular Disease, Institute of Health Research (INCLIVA), Valencia, Spain. Dr Servera is also affiliated with the Department of Physical Therapy, and Dr Marin is affiliated with the Department of Medicine, Universitat de Valencia, Valencia, Spain.
During these episodes, reduced cough effectiveness and impaired alveolar ventilation can have fatal consequences for patients with ALS. ${ }^{4}$ Therefore, during the management of respiratory problems in ALS, it is important to recognize that those patients in a medically stable condition could be at risk of developing an ineffective cough or respiratory failure during an acute respiratory infection.

It has been demonstrated that, in medically stable patients with ALS with an apparently effective cough capac-

\footnotetext{
Dr Bañuls was supported by a grant from the Institute of Health Research (INCLIVA) sponsored by VitalAire. The other authors have disclosed no conflicts of interest.
}

Correspondence: Jesus Sancho MD, Respiratory Care Unit, Respiratory Medicine Department, Hospital Clinico Universitario, Avenida Blasco Ibañez 17, 46010 Valencia, Spain. E-mail: jesus.sancho@uv.es.

DOI: $10.4187 /$ respcare. 03553 
ity, measurement of bulbar dysfunction and peak cough flow can predict spontaneous cough ineffectiveness during an acute lower respiratory tract infection. ${ }^{5}$ The aim of this study was to determine whether a clinical or functional parameter can predict the failure of respiratory muscles to

\section{See the Related Editorial on Page 625}

maintain adequate ventilation during an acute lower respiratory tract infection in medically stable, non-ventilated subjects with ALS.

\section{Methods}

We performed a prospective study from January 2004 to June 2012 in a respiratory care unit of a university hospital that included all subjects with ALS who, according to the criteria of the American College of Chest Physicians, ${ }^{6}$ did not require noninvasive ventilation (NIV) at home and who were admitted to the respiratory care unit due to an acute lower respiratory tract infection. The American College of Chest Physicians established that NIV should be initiated in the presence of symptoms related to hypoventilation with at least one physiologic criterion: $\mathrm{P}_{\mathrm{aCO}_{2}}$ of $>45 \mathrm{~mm} \mathrm{Hg}$, nocturnal oxygen desaturation for 5 consecutive min, FVC of $<50 \%$ of predicted, and maximum inspiratory pressure of $>-60 \mathrm{~cm} \mathrm{H}_{2} \mathrm{O}$. Exclusion criteria were refusal to participate or the presence of previous pulmonary or airway disease. All subjects had to be in a medically stable condition for at least 1 month previous to the acute episode. Informed consent was obtained from each subject who took part in the study, and the study protocol was approved by the hospital's ethics committee.

In accordance with the European Respiratory Society guidelines, ${ }^{7}$ subjects were considered to be suffering from an acute lower respiratory tract infection in the presence of a cough with at least one or more of the following criteria: sputum production, dyspnea, wheezing, or chest discomfort. Acute bronchitis was diagnosed as an acute illness occurring in a patient without chronic lung disease, with symptoms including cough and associated with other symptoms or clinical signs that suggest lower respiratory tract infection. Pneumonia was diagnosed when, in addition to the previous criteria, a chest $\mathrm{x}$-ray revealed lung shadowing that was likely to be new.

\section{Clinical and Functional Impairment Assessment}

The diagnosis of ALS was carried out in accordance with the revised El Escorial criteria. ${ }^{8}$ Spirometry was assessed with a pneumotachograph spirometer (MS 2000, Schatzman, Madrid, Spain) in accordance with the European Respiratory Society's guidelines ${ }^{9}$ as described previously. ${ }^{5}$ Maximum in-

\section{QUICK LOOK}

\section{Current knowledge}

Patients with amyotrophic lateral sclerosis (ALS) suffer progressive respiratory muscle weakness, leading to ventilatory failure and ineffective cough and concluding in morbidity and mortality. Acute respiratory tract infections in these patients can exacerbate muscle weakness and trigger acute respiratory failure.

\section{What this paper contributes to our knowledge}

Medically stable, non-ventilated ALS subjects with an FVC of $<55 \%$ of predicted and a peak cough flow of $<2.9 \mathrm{~L} / \mathrm{s}$ had a high probability of developing respiratory failure. In this population, ventilatory support is frequently required during an acute lower respiratory tract infection.

spiratory and maximum expiratory pressures were measured (Electrometer 78.905A, Hewlett-Packard, Palo Alto, California) according to the technique of Black and Hyatt. ${ }^{10}$ Cough capacity was assessed with the MS 2000 pneumotachograph spirometer as described previously. ${ }^{5}$ Peak cough flow, maximum insufflation capacity, manually assisted peak cough flow, and mechanically assisted peak cough flow were recorded. Functional impairment was evaluated using the revised Amyotrophic Lateral Sclerosis Functional Rating Scale, ${ }^{11}$ and bulbar involvement was assessed in accordance with the Norris scale bulbar subscore. ${ }^{12}$

\section{Follow-up Protocol and Respiratory Muscle Aids During Acute Episodes}

After inclusion in the study, subjects received a scheduled clinical and functional assessment by a pneumologist every 3 months. When peak cough flow levels were found to be $<4.25 \mathrm{~L} / \mathrm{s}$, mechanically assisted cough was prescribed. 5 Subjects were trained to use mechanically assisted cough every $8 \mathrm{~h}$ and whenever the subject had a feeling of retained respiratory secretions. ${ }^{1}$

Subjects were encouraged to come to our respiratory department at any time if they suffered dyspnea, difficulty in intrathoracic secretion clearance, audible thoracic respiratory sounds, or fever. In these cases, after a medical evaluation, subjects were considered for hospitalization at the respiratory care unit if they met the next criteria: presence of respiratory failure, need for ventilatory support (invasive or noninvasive), need for intensive mechanically assisted cough ( $>4$ sessions/h), or need for intravenous medication.

During an acute episode in our respiratory care unit, ventilatory support was provided in the presence of one or 
Table 1. Demographic Data and Pulmonary Function Parameters of Non-Ventilated Subjects With ALS Admitted Due to a Lower Respiratory Tract Infection

\begin{tabular}{|c|c|c|c|}
\hline & With NIV $(n=15)$ & Without NIV $(n=17)$ & $P$ \\
\hline Age, y & $62.6 \pm 8.4$ & $59.2 \pm 12.1$ & .36 \\
\hline Males/females & $6 / 9$ & $7 / 10$ & .95 \\
\hline BMI, $\mathrm{kg} / \mathrm{m}^{2}$ & $25.2 \pm 3.7$ & $25.6 \pm 2.7$ & .74 \\
\hline Time from ALS onset, mo & $36.4 \pm 30.8$ & $31.6 \pm 15.1$ & .58 \\
\hline ALS onset (spinal/bulbar), $n$ & $8 / 7$ & $15 / 2$ & .07 \\
\hline ALSFRS-R & $19.3 \pm 8.9$ & $26.3 \pm 7.6$ & .04 \\
\hline NBS & $17.9 \pm 12.9$ & $28.0 \pm 9.6$ & .02 \\
\hline FVC, L & $1.19 \pm 0.55$ & $1.88 \pm 0.90$ & .02 \\
\hline FVC, $\%$ predicted & $43.1 \pm 20.3$ & $66.4 \pm 20.3$ & $<.001$ \\
\hline Maximum insufflation capacity, L & $1.47 \pm 0.72$ & $2.19 \pm 1.00$ & .03 \\
\hline Peak cough flow, L/s & $2.4 \pm 1.3$ & $4.2 \pm 1.68$ & $<.001$ \\
\hline Manually assisted peak cough flow, L/s & $2.7 \pm 1.5$ & $4.7 \pm 1.9$ & $<.001$ \\
\hline Mechanically assisted peak cough flow, L/s & $3.2 \pm 0.9$ & $4.0 \pm 0.8$ & .01 \\
\hline $\mathrm{P}_{\text {Imax }}, \mathrm{cm} \mathrm{H}_{2} \mathrm{O}$ & $-37.6 \pm 27.7$ & $-54.9 \pm 20.2$ & .07 \\
\hline $\mathrm{P}_{\text {Emax }}, \mathrm{cm} \mathrm{H}_{2} \mathrm{O}$ & $61.1 \pm 32.8$ & $81.7 \pm 35.0$ & .14 \\
\hline $\begin{array}{l}\text { Values are provided as mean } \pm \text { SD unless indicated otherwi } \\
\text { ALS }=\text { amyotrophic lateral sclerosis } \\
\text { NIV }=\text { noninvasive ventilation } \\
\text { BMI }=\text { body mass index } \\
\text { ALSFRS-R }=\text { revised Amyotrophic Lateral Sclerosis Functi } \\
\text { NBS }=\text { Norris scale bulbar subscore } \\
P_{\text {Imax }}=\text { maximum inspiratory pressure } \\
P_{\text {Emax }}=\text { maximum expiratory pressure }\end{array}$ & & & \\
\hline
\end{tabular}

more of the following clinical and/or physiologic criteria: severe dyspnea, lethargy, use of respiratory accessory muscles, $\mathrm{P}_{\mathrm{aCO}_{2}}$ of $>45 \mathrm{~mm} \mathrm{Hg}, \mathrm{P}_{\mathrm{aO}_{2}}$ of $<60 \mathrm{~mm} \mathrm{Hg}$, or $\mathrm{pH}$ of $<7.35$. Initially, mechanical ventilation was provided noninvasively. NIV was delivered via volume control continuous mandatory ventilation (PV 501, PV 403, and Vivo 50, Breas Medical, Mölndal, Sweden; AiroxHome2 and Legendair, Airox, Pau Cedex, France) as described previously. ${ }^{4}$

Respiratory secretions were managed with mechanically assisted cough (CoughAssist, Philips Respironics, Murrysville, Pennsylvania) through an oronasal mask (Martin Vecino, Madrid, Spain) at least twice every $8 \mathrm{~h}$ and whenever $\mathrm{S}_{\mathrm{pO}_{2}}$ decreased, the ventilator peak inspiratory pressure increased, or the subject had an increase in dyspnea or the sensation of retained secretions. It was often used as frequently as every 5-10 min. As the subject's clinical status improved, the frequency of the mechanically assisted cough sessions was decreased

If the subject had previously agreed to it, endotracheal intubation was considered when NIV could not ameliorate hypercapnia or relieve respiratory distress, lethargy, or rapidly decreasing $\mathrm{S}_{\mathrm{PO}_{2}}$; when mechanically assisted cough was unable to adequately remove airway secretions; and/or when subjects felt continuously encumbered or dyspneic. In those subjects who refused endotracheal intubation, palliative therapy was provided.

\section{Statistical Analysis}

The data used in the study were those measured at a subject's previously scheduled assessment (in a medically stable condition). Data were expressed as mean \pm SD. Data comparisons were performed using the Student $t$ test. Categorical data were compared using the chi-square test. Univariate and multivariate logistic regression analyses were used to determine those variables that were independently associated with the need for NIV during the acute episode. Receiver operating characteristic curves were used to identify a cutoff point in those variables that best predicted the subjects who needed NIV during an acute episode. The level for statistical significance was taken as $P<.05$.

\section{Results}

During the study period, 32 non-ventilated subjects with ALS were admitted to our respiratory care unit due to an acute lower respiratory infection. All subjects agreed to participate in the study, and none fulfilled the exclusion criteria. Data on demographics, respiratory function, and cough capacity assessment of the subjects included in the study are shown in Table 1. All subjects were in a medically stable condition, without symptoms of hypoventilation or nocturnal desaturations, and all were normocapnic 
Table 2. Arterial Blood Gases at Hospital Admission Without Supplementary Oxygen

\begin{tabular}{lccc}
\hline \hline & With NIV & $\begin{array}{c}\text { Without } \\
\text { NIV }\end{array}$ & $P$ \\
\hline $\mathrm{pH}$ & $7.36 \pm 0.11$ & $7.41 \pm 0.4$ & .20 \\
$\mathrm{P}_{\mathrm{OO}_{2}}, \mathrm{~mm} \mathrm{Hg}$ & $58 \pm 17$ & $72 \pm 15$ & .06 \\
$\mathrm{P}_{\mathrm{CO}_{2}}, \mathrm{~mm} \mathrm{Hg}$ & $59 \pm 24$ & $37 \pm 6$ & .01 \\
$\mathrm{HCO}_{3}^{-}, \mathrm{mmol} / \mathrm{L}$ & $27 \pm 2$ & $25 \pm 1$ & .06 \\
$\mathrm{~S}_{\mathrm{aO}}, \%$ & $87 \pm 7$ & $93 \pm 6$ & .04 \\
& & & \\
Values are provided as mean $\pm \mathrm{SD} ._{\mathrm{NIV}=\text { noninvasive ventilation }}$ & & \\
$\mathrm{S}_{\mathrm{aO}}=$ arterial oxygen saturation & & & \\
\hline
\end{tabular}

and had no rapid decline in FVC for the previous 6 months. Seven subjects $(21.2 \%)$ were using mechanically assisted cough. Causes of hospital admission were acute bronchitis $(72.7 \%)$, community-acquired pneumonia $(24.2 \%)$, and aspiration pneumonia $(3.0 \%)$.

A total of 15 subjects (45.5\%) needed ventilatory support during the acute respiratory infection; mechanical ventilation was initially provided in the form of NIV in every case. Statistical differences were found between those subjects who needed NIV and those who did not in previous measurements of FVC, percent-of-predicted FVC, maximum insufflation capacity, peak cough flow, manually assisted peak cough flow, mechanically assisted peak cough flow, revised Amyotrophic Lateral Sclerosis Functional Rating Scale scores, and Norris scale bulbar subscores (see Table 1).

Those subjects who needed NIV upon hospital admission presented with higher $\mathrm{P}_{\mathrm{aCO}}$ (Table 2) During the acute episode, 26 subjects $(81.25 \%)$ required mechanically assisted cough due to ineffective cough capacity. Those subjects who did not need NIV were managed successfully and discharged home. Of those subjects who required NIV during the acute episode, 11 subjects (73.3\%) were successfully managed noninvasively, but 2 subjects $(13.3 \%)$ underwent endotracheal intubation and mechanical ventilation, and 2 subjects (13.3\%) who refused invasive management received full palliative medical care. No statistical differences were found between those who needed NIV and those who did not in mean hospital stay $(7.58 \pm 4.90$ vs $8.36 \pm 3.98 \mathrm{~d}, P=.68)$

In the univariate logistic regression analysis, the variables that most accurately predicted the need for NIV during an acute respiratory tract infection in non-ventilated subjects with ALS were FVC, percent-of-predicted FVC, peak cough flow, manually assisted peak cough flow, mechanically assisted peak cough flow, and Norris scale bulbar subscores (Table 3). In the multivariate logistic regression analysis, the only variables that predicted the need for
Table 3. Predictors of Need for NIV During an Acute Episode in Non-Ventilated Subjects With ALS

\begin{tabular}{lccc}
\hline \hline \multicolumn{1}{c}{ Parameter } & Odds Ratio & $95 \%$ CI & $P$ \\
\hline Sex & 0.95 & $0.23-3.88$ & .95 \\
Age & 0.97 & $0.90-1.04$ & .35 \\
Onset & 4.37 & $0.88-21.71$ & .07 \\
Time from ALS onset & 0.99 & $0.96-1.02$ & .57 \\
FVC & 4.67 & $1.13-19.21$ & .03 \\
FVC, \% predicted & 1.06 & $1.01-1.11$ & .01 \\
Maximum insufflation capacity & 2.93 & $1.00-8.61$ & .05 \\
Peak cough flow & 2.39 & $1.23-4.66$ & .01 \\
Manually assisted peak cough flow & 1.99 & $1.15-3.47$ & .01 \\
Mechanically assisted peak cough flow & 3.04 & $1.16-7.94$ & .02 \\
$\mathrm{P}_{\text {Imax }}$ & 0.96 & $0.93-1.00$ & .08 \\
$\mathrm{P}_{\text {Emax }}$ & 1.02 & $0.99-1.05$ & .14 \\
ALSFRS-R & 1.12 & $1.00-1.25$ & .06 \\
NBS & 1.08 & $1.01-1.16$ & .03 \\
BMI & 1.05 & $0.80-1.38$ & .73 \\
& & & \\
\hline Univariate analysis & & & \\
NIV = noninvasive ventilation & & & \\
PLS = amyotrophic lateral sclerosis & & & \\
$P_{\text {Emax }}=$ maximum inspiratory pressure & & & \\
ALSFRS-R = revised Amyotrophic Lateral Sclerosis Functional Rating Scale & & \\
NBS = Norris scale bulbar subscore & & & \\
BMI = body mass index & & & \\
\hline & & & \\
\hline
\end{tabular}

NIV were percent-of-predicted FVC (odds ratio [OR] of $1.06,95 \%$ CI 1.01-1.11, $P=.02$ ) and peak cough flow (OR 2.57, 95\% CI $1.18-5.59, P=.02$ ). In the receiver operating characteristic curve analysis, the variables with the highest area under the curve were percent-of-predicted FVC (area under the curve of 0.79 , 95\% CI $0.63-0.94$, $P=.01$ ) and peak cough flow (area under the curve 0.81 ; $95 \%$ CI $0.66-0.96, P=.00)$ Cutoff points of $55 \%$ for percent-of-predicted FVC (sensitivity of 0.72 , specificity of 0.78 , positive predictive value of 0.81 , negative predictive value of 0.68 ) and $2.9 \mathrm{~L} / \mathrm{s}$ for peak cough flow (sensitivity of 0.77 , specificity of 0.71 , positive predictive value of 0.77 , negative predictive value of 0.71 ) were the best predictors for the identification of non-ventilated subjects with ALS who required NIV during acute respiratory episodes.

\section{Discussion}

The findings of this study show that, in non-ventilated patients with ALS in a medically stable condition, the measurement of percent-of-predicted FVC and peak cough flow may be able to predict the need for ventilatory support during possible lower respiratory tract infections. Compared with normal subjects, neuromuscular disease patients present a reduction in respiratory muscle strength during a respiratory infection, changing an ordinary chest 
cold into a life-threatening situation. ${ }^{13,14}$ During these acute episodes, the weakness of inspiratory muscles can lead to a decrease in alveolar ventilation capacity and can produce inadequate lung expansion with microatelectasis and retained secretions, leading to a ventilation/perfusion mismatch with hypoxemia. ${ }^{14}$ Furthermore, bronchial inflammation and an increase in the quantity and purulence of respiratory secretions or secretion encumbrance due to a decrease in cough capacity lead to an increase in the ventilatory load. This imbalance between the ventilatory load and respiratory muscle strength can trigger acute respiratory failure and make it necessary for ventilatory assistance to be provided. In such situations, NIV has proven able to provide adequate ventilation and thus avoid endotracheal intubation. ${ }^{4}$

In this way, some patients with ALS in a medically stable condition who have not yet needed home NIV because their respiratory muscles have been able to maintain adequate ventilation can develop acute respiratory failure during an acute lower respiratory infection and thus require ventilatory assistance to support life. ${ }^{13,14}$ The results obtained in this study help to establish, for patients in a stable condition for whom home NIV is not indicated, the point at which there is a significant risk that their respiratory muscles may be unable to provide effective ventilation during an acute respiratory infection.

Despite its lower sensitivity in detecting early diaphragmatic weakness, FVC is the most widely used variable for the evaluation of respiratory function in patients with ALS, and its regular measurement is recommended. ${ }^{15}$ Percent-of-predicted FVC and the slope of decline of percent-of-predicted FVC have been significantly correlated with ALS survival. ${ }^{16}$ An FVC of $<50 \%$ of predicted is one of the criteria for commencement of NIV in ALS, although recent studies, with important bias, have proposed an earlier intervention (FVC of $>65 \%$ of predicted) with NIV. ${ }^{17,18}$ However, recent guidelines recommend the commencement of NIV in the presence of symptoms of hypoventilation, hypercapnia, or rapid reduction in FVC despite a fixed percent-of-predicted FVC. ${ }^{19}$ The findings of this study show that a baseline FVC of $<55 \%$ of predicted in a stable condition suggests ineffective ventilatory capacity during acute chest episodes. A reduced FVC in patients with ALS is related to respiratory muscle weakness; however, a poor correlation between FVC and symptoms, respiratory muscle strength, and sleep-related breathing disorders has been described. ${ }^{20,21} \mathrm{FVC}$ may not be reduced until there is severe muscle weakness, as a consequence of the sigmoid relationship of the lung pressure-volume curves being unable to detect modest falls in muscle strength. ${ }^{22}$ Thus, patients with respiratory muscle weakness may have an FVC of $>50 \%$ of predicted. There were subjects in our study who had a certain degree of respiratory muscle weakness despite not having symptoms related to hypoventilation or even hypercapnia in a medically stable condition. During respiratory tract infections, reductions in respiratory muscle strength in the range of $10-15 \mathrm{~cm} \mathrm{H}_{2} \mathrm{O}$ have been reported and, as a consequence, decreases in FVC of up to $7 \% .{ }^{23}$ When associated with an increased load on the respiratory system, this can precipitate hypercapnic respiratory failure. ${ }^{24}$ Thus, our results show that a decreased cough capacity measured with peak cough flow can precipitate respiratory failure due to an increased ventilator load secondary to retained secretions; this fact exposes the need for adequate respiratory secretion management during the acute episode.

Previous studies on patients with other neuromuscular diseases have reported predictors of respiratory failure and the need for mechanical ventilation during an acute episode based on the measurement of daily bedside respiratory parameters during a hospital stay. In Guillain-Barré syndrome, the presence of bulbar dysfunction (OR 17.5, $95 \%$ CI 5.2-59.1) and a vital capacity of $<20 \mathrm{~mL} / \mathrm{kg}$ (OR $15.0,95 \%$ CI $4.1-54.5$ ) have been identified as the strongest predictors of respiratory failure. ${ }^{25}$ However, in myasthenia gravis, the serial measurement of vital capacity is a poor predictor of the need for ventilatory support due to the unpredictable and erratic course of this disease. ${ }^{26}$ In myasthenic crisis precipitated by surgery, myasthenia lasting for $>6 \mathrm{y}$, a history of chronic respiratory disease, a dose of pyridostigmine, and a preoperative vital capacity of $<2.9 \mathrm{~L}$ have been singled out as predictors of the need for postoperative mechanical ventilation. ${ }^{27}$

Our results could provide support for the proposed early use of NIV in patients with ALS. However, the lack of improvement perceived by the patient at home, such as with regard to the relief of hypoventilation-related symptoms, can interfere with NIV tolerance. For all of the subjects enrolled in this study, while they were in a medically stable condition at home, they had no symptoms of hypoventilation, and all of them were normocapnic. Perhaps a more practical approach would be for those non-ventilated ALS patients with an FVC of $<55 \%$ of predicted to become familiar with NIV in case they need it during an acute respiratory episode and for patient training with this technique to begin. Moreover, the hospital staff should receive this information during exacerbations, so they can provide appropriate respiratory care (in accordance with the patient's previous directives) without delays. In this way, international guidelines recommend that a respiratory insufficiency management plan be established for patients with ALS before respiratory complications occur. ${ }^{28}$

The small number of subjects included in this study represents a limitation when assessing the value of the results. However, we included all subjects with ALS treated in a specialist referral unit over a period of $8 \mathrm{y}$ who met the admission criteria of the protocol. The fact that only subjects from a single center were included can be seen as a positive factor, as this ensures compliance with the protocol and serves to avoid other confounding factors. 


\section{Predicting NIV for SubJects With ALS}

\section{Conclusions}

In summary, non-ventilated patients with ALS in a medically stable condition with an FVC of $<55 \%$ of predicted and a peak cough flow of $<2.9 \mathrm{~L} / \mathrm{s}$ are more likely to develop respiratory failure, which will require ventilatory support during an acute lower respiratory tract infection.

\section{REFERENCES}

1. Bourke SC, Tomlinson M, Williams TL, Bullock RE, Shaw PJ, Gibson GJ. Effects of noninvasive ventilation on survival and quality of life in patients with amyotrophic lateral sclerosis: a randomized controlled trial. Lancet Neurol 2006;5(2):140-147.

2. Lechtzin N, Wiener CM, Clawson L, Chaudhry V, Diette GB. Hospitalization in amyotrophic lateral sclerosis: causes, costs and outcomes. Neurology 2001;56(6):753-757.

3. Ambrosino N, Carpenè N, Gherardi M. Chronic respiratory care for neuromuscular diseases in adults. Eur Respir J 2009;34(2):444-451.

4. Servera E, Sancho J, Zafra MJ, Catalá A, Vergara P, Marín J. Alternatives to endotracheal intubation for patients with neuromuscular diseases. Am J Phys Med Rehabil 2005;84(11):851-857.

5. Sancho J, Servera E, Díaz J, Marín J. Predictors of ineffective cough during a chest infection in patients with stable amyotrophic lateral sclerosis. Am J Respir Crit Care Med 2007;175(12):1266-1271.

6. Clinical indications for noninvasive positive pressure ventilation in chronic respiratory failure due to restrictive lung disease, COPD and nocturnal hypoventilation-a consensus conference report. Chest 1999; 116(2):521-534

7. Woodhead M, Blasi F, Ewig S, Garau J, Huchon G, Ieven M, et al. Guidelines for the management of adult lower respiratory tract infections. Clin Microbiol Infect 2011;17(Suppl 6): E1-E59.

8. Brooks BR, Miller RG, Swash M, Munsat TL, World Federation of Neurology Research Group on Motor Neuron Diseases. El Escorial revisited: revised criteria for the diagnosis of amyotrophic lateral sclerosis. Amyotroph Lateral Scler Other Motor Neuron Disord 2000;1(5): 293-299.

9. Miller MR, Hankinson J, Brusasco V, Burgos F, Casaburi R, Coates A, et al. Standardisation of spirometry. Eur Respir J 2005;26(2):319-338.

10. Black LF, Hyatt RE. Maximal respiratory pressures: normal values and relationship to age and sex. Am Rev Respir Dis 1969;99(5):696-702.

11. Cedarbaum JM, Stambler N, Malta E, Fuller C, Hilt D, Thurmond B, Nakanishi A. The ALSFRS-R: a revised ALS function ranting scale that incorporates assessment of respiratory function. BDNF ALS Study Group (Phase III). J Neurol Sci 1999;169(1-2):13-21.

12. Lacomblez L, Bouche P, Bensimon G, Meininger V. A double-blind, placebo-controlled trial of high doses of gangliosides in amyotrophic lateral sclerosis. Neurology 1989;39(12):1635-1637.

13. Poponick JM, Jacobs I, Supinski G, DiMarco AF. Effect of upper respiratory track infection in patients with neuromuscular diseases. Am J Respir Crit Care Med 1997;156(2 Pt 1):659-664.
14. Servera E, Sancho J, Gómez-Merino E, Briones ML, Vergara P, Pérez D, Marín J. Noninvasive management of an acute chest infection for a patient with ALS. J Neurol Sci 2003;209(1-2):111-113.

15. Miller RG, Jackson CE, Kasarskis EJ, England JD, Forshew D, Johnston W, et al. Practice parameter update. The care of the patient with amyotrophic lateral sclerosis: drug, nutritional and respiratory therapies (an evidenced based review): report of the Quality Standards Subcommittee of the Academy of Neurology. Neurology 2009; 73(15):1218-1226.

16. Clavelou P, Blanquet M, Peyrol F, Ouchchane L, Gerbaud L. Rates of progression of weight and forced vital capacity as relevant measurement to adapt amyotrophic lateral sclerosis management for patient. Result of a French multicenter cohort survey. J Neurol Sci 2013;331(1-2):126-131.

17. Lechtzin N, Scott Y, Busse AM, Clawson LL, Kimball R, Wiener $\mathrm{CM}$. Early use of noninvasive ventilation prolongs survival in subject with ALS. Amyotroph Lateral Scler 2007;8(3):185-188.

18. Carratu P, Spicuzza L, Cassano A, Maniscalco M, Gadaleta F, Lacedonia D, et al. Early treatment with noninvasive positive pressure ventilation prolongs survival in amyotrophic lateral sclerosis patients with nocturnal respiratory insufficiency. Orphanet J Rare Dis 2009;4:10.

19. Farrero E, Antón A, Egea CJ, Almaraz MJ, Masa JF, Utrabo I, et al. Guidelines for the management of respiratory complications in patients with neuromuscular disease. Arch Bronconeumol 2013;49(7):306-313.

20. Jackson CE, Rosenfeld J, Moore DH, Brian WW, Barohn RJ, Wrench $\mathrm{M}$, et al. A preliminary evaluation of a prospective study of pulmonary function studies and symptoms of hypoventilation in ALS/MND patients. J Neurol Sci 2001;191(1-2):75-78.

21. Lyall RA, Donalson N, Polkey MI, Leigh PN, Moxham J. Respiratory muscle strength and ventilator failure in amyotrophic lateral sclerosis. Brain 2001;124(Pt 10):2000-2013.

22. De Troyer A, Borenstein S, Cordier R. Analysis of lung volume restriction in patients with respiratory muscle weakness. Thorax 1980; 35(8):603-610.

23. Mier-Jedrzejowicz A, Brophy C, Green M. Respiratory muscle weakness during upper respiratory tract infections. Am Rev Respir Dis 1988;138(1):5-7.

24. Laroche CM, Moxham J, Green M. Respiratory muscle weakness and fatigue. Q J Med 1989;71(265):373-397.

25. Lawn ND, Fletcher DD, Henderson RD, Wolter TD, Wijdicks EF. Anticipating mechanical ventilation in Guillain-Barré syndrome. Arch Neurol 2001;58(6):893-898.

26. Rieder P, Louis M, Jolliet P, Chevrolet JC. The repeated measurement of vital capacity is a poor predictor of the need for mechanical ventilation in myasthenia gravis. Intensive Care Med 1995;21(8):663-668.

27. Leventhal SR, Orkin FK, Hirsh RA. Prediction of the need for postoperative mechanical ventilation in myasthenia gravis. Anesthesiology 1980;53(1):26-30.

28. EFNS Task Force on Diagnosis and Management of Amyotrophic Lateral Sclerosis, Andersen PM, Abrahams S, Borasio GD, de Carvalho M, Chio A, et al. EFNS guidelines on the clinical management of amyotrophic lateral sclerosis (MALS)-revised report of an EFNS task force. Eur J Neurol 2012;19(3):360-375.

This article is approved for Continuing Respiratory Care Education credit. For information and to obtain your CRCE

(free to AARC members) visit

www.rcjournal.com

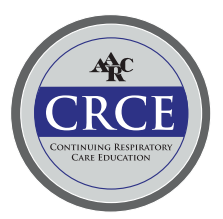

